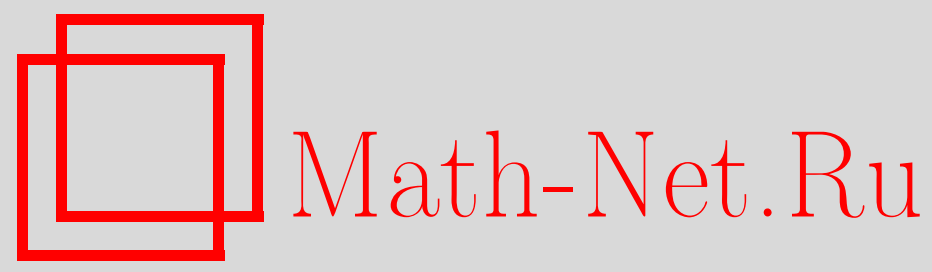

А. Ю. Александров, Об устойчивости решений нелинейных систем с неограниченными возмущениями, Матем. заметкu, 1998, том 63, выпуск 1, 3-8

DOI: https://doi.org/10.4213/mzm1242

Использование Общероссийского математического портала Math-Net.Ru подразумевает, что вы прочитали и согласны с пользовательским соглашением http://www . mathnet.ru/rus/agreement

Параметры загрузки:

IP : 3.85 .73 .92

26 апреля 2023 г., $12: 38: 38$

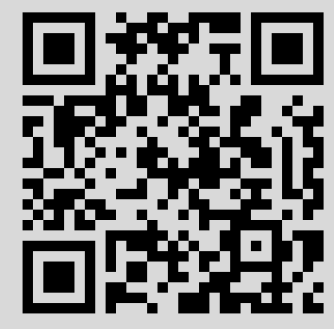




\section{ОБ УСТОЙЧИВОСТИ РЕШЕНИЙ НЕЛИНЕЙНЫХ СИСТЕМ С НЕОГРАНИЧЕННЫМИ ВОЗМУЩЕНИЯМИ}

\section{А. Ю. Александров}

Исследуются системы дифференциальных уравнений с возмущениями, являющимися неограниченными функциями времени. Предложен метод построения функций Ляпунова для определения условий, при выполнении которых возмущения не нарушают асимптотическую устойчивость решений рассматриваемых систем.

Библиография: 8 названий.

1. В настоящей работе исследуется воздействие нестационарных возмущений на устойчивость решений систем нелинейных дифференциальных уравнений. При этом предполагается, что возмущения являются неограниченньми функциями времени с определенной степенью роста.

Рассмотрим систему

$$
\dot{x}_{s}=f_{s}(X), \quad s=1, \ldots, n .
$$

Здесь $f_{s}(X)$ - непрерывно-дифференцируемые однородные функции порядка $\mu$, где $\mu-$ рациональное число с нечетньм знаменателем, $\mu>1$. Будем предполагать, что нулевое решение системы (1) асимптотически устойчиво.

Наряду с системой (1) рассмотрим возмущенную систему

$$
\dot{x}_{s}=f_{s}(X)+r_{s}(t, X), \quad s=1, \ldots, n,
$$

где функции $r_{s}(t, X)$ определены и непрерывны в области

$$
t \geqslant 0, \quad\|X\|<H, \quad H \geqslant 0,
$$

и удовлетворяют условиям, гарантирующим в этой области существование и единственность решений системы (2), непрерывно зависящих от начальных данных. Пусть система (2) также имеет нулевое решение. Исследуем вопрос, при каких условиях возмущения не нарушают асимптотическую устойчивость нулевого решения.

Теоремы об устойчивости по нелинейному приближению были получены в работах И.Г. Малкина и Н.Н. Красовского [1], [2]. При этом предполагалось, что возмущения ограничены в области (3). Известно (см. [2]), что если функции $r_{s}(t, X)$ в области (3) удовлетворяют неравенствам

$$
\left|r_{s}(t, X)\right| \leqslant D\|X\|^{\sigma}, \quad s=1, \ldots, n,
$$

где $D$ и $\sigma$-положительные постоянные, причем $\sigma>\mu$, то нулевое решение системы (2) асимптотически устойчиво.

В настоящей работе рассмотрим случай, когда возмушения являются неограниченными функциями времени. 
2. Пусть в области (3) вьполнены неравенства

$$
\left|r_{s}(t, X)\right| \leqslant D(t+1)^{\alpha}\|X\|^{\sigma}, \quad s=1, \ldots, n,
$$

ограничивающие степень роста возмущений. Здесь $D, \sigma$ и $\alpha$ - положительные постоянные.

Определим, насколько порядок возмущений должен превьшшать порядок функций $f_{s}(X)$, чтобы из асимптотической устойчивости нулевого решения системы (1) следовала асимптотическая устойчивость нулевого решения системы (2).

ТЕОРЕМА 1. При выполнении неравенства

$$
\sigma>\alpha(\mu-1)+\mu
$$

нулевое решение системы (2) асимптотически устойчиво.

ДоКАЗАТЕЛЬСТво. Известно [3, с. 115-123], что из асимптотической устойчивости нулевого решения системы (1) следует существование функций $V(X)$ и $W(X)$, обладающих свойствами:

1) $V(X)$ и $W(X)$ - положительно определенные функции;

2) $V(X)$ и $W(X)$ - однородные функции порядка $m$ и $m+\mu-1$ соответственно, $m>1$;

3) функция $V(X)$ непрерьвно- дифференцируема;

4) имеет место равенство

$$
\sum_{s=1}^{n} \frac{\partial V}{\partial x_{s}} f_{s}(X)=-W(X)
$$

В работе [2] доказано, что решения $X\left(t, X_{0}, t_{0}\right)$ системы (1) удовлетворяют при $t \geqslant t_{0}$ неравенствам

$$
\left\|X\left(t, X_{0}, t_{0}\right)\right\|^{\mu-1} \leqslant\left(c_{1}\left\|X_{0}\right\|^{1-\mu}+c_{2}\left(t-t_{0}\right)\right)^{-1},
$$

где $c_{1}$ и $c_{2}$ - положительные постоянные.

Покажем, что существуют положительные числа $\gamma, A$ и $T$ такие, что для любого решения $X(t)=X\left(t, X_{0}, t_{0}\right)$ системы $(2)$, начальные данные которого удовлетворяют условиям

$$
t_{0} \geqslant T, \quad\left\|X_{0}\right\|^{\mu-1}<\frac{\gamma}{t_{0}}
$$

при всех $t \geqslant t_{0}$ справедливо неравенство

$$
\|X(t)\|^{\mu-1}<\frac{A}{t} .
$$

Для однородных функций $V(X)$ и $W(X)$ при всех $X \in E^{n}$ имеют место неравенства

$$
\begin{gathered}
a_{1}\|X\|^{m} \leqslant V(X) \leqslant a_{2}\|X\|^{m}, \quad\left|\frac{\partial V(X)}{\partial x_{s}}\right| \leqslant a_{3}\|X\|^{m-1}, \quad s=1, \ldots, n, \\
b_{1}\|X\|^{m+\mu-1} \leqslant W(X) \leqslant b_{2}\|X\|^{m+\mu-1},
\end{gathered}
$$

где $a_{1}, a_{2}, a_{3}, b_{1}, b_{2}$ - положительные постоянные (см. [3]). 
Выберем числа $\gamma, A$ и $T$ так, чтобы вьполнялись условия

$$
\begin{gathered}
2 m a_{2}>(\mu-1) b_{1} \gamma, \quad \gamma<A, \quad(\mu-1) b_{1} a_{1}^{(\mu-1) / m} A>2 m a_{2}^{1+(\mu-1) / m}, \quad \frac{A}{T}<H^{\mu-1}, \\
2 n D a_{3} A^{(\sigma-\mu) /(\mu-1)}(t+1)^{\alpha} t^{-(\sigma-\mu) /(\mu-1)}<b_{1} \quad \text { при } t \geqslant T .
\end{gathered}
$$

Рассмотрим решение $X(t)$ системы (2) с начальными данньми, удовлетворяющими неравенствам (5). Предположим, что существует момент времени $t_{1}>t_{0}$ такой, что

$$
\left\|X\left(t_{1}\right)\right\|^{\mu-1}=\frac{A}{t_{1}}
$$

а при $t \in\left[t_{0}, t_{1}\right)$ справедливо неравенство (6). Продифференцируем функцию $V(X)$. В силу системы $(2)$ при $t \in\left[t_{0}, t_{1}\right]$ имеем

$$
\begin{aligned}
\frac{d V(X(t))}{d t} & =-W(X(t))+\sum_{s=1}^{n} \frac{\partial V(X(t))}{\partial x_{s}} r_{s}(t, X(t)) \\
& \leqslant-b_{1}\|X(t)\|^{m+\mu-1}+n D a_{3}(t+1)^{\alpha}\|X(t)\|^{m+\sigma-1}<-\frac{b_{1}}{2}\|X(t)\|^{m+\mu-1} .
\end{aligned}
$$

Применяя метод оценок (см. [3], [4]), приходим к неравенству

$$
t_{1}\left(\frac{(\mu-1) b_{1}}{2 m a_{2}}-\frac{1}{A}\left(\frac{a_{2}}{a_{1}}\right)^{(\mu-1) / m}\right) \leqslant t_{0}\left(\frac{(\mu-1) b_{1}}{2 m a_{2}}-\frac{1}{\gamma}\right) .
$$

Из условий выбора чисел $\gamma, A$ и $T$ следует, что левая часть данного неравенства положительна, а правая - отрищательна. Получаем противоречие.

Таким образом, если вьполнены неравенства (5), то при всех $t \geqslant t_{0}$ для решения $X(t)$ справедлива оценка (6).

Используя доказанное свойство решений системы (2), а также их непрерывную зависимость от начальных данных, получаем, что нулевое решение системы (2) асимптотически устойчиво. Теорема доказана.

3. Далее будем предполагать, что возмущенная система (2) представима в виде

$$
\dot{x}_{s}=f_{s}(X)+\sum_{j=1}^{k} b_{s j}(t) h_{j}(X), \quad s=1, \ldots, n .
$$

Здесь $h_{j}(X)$ - непрерьвно- дифференцируемые однородные функции порядка $\sigma$, где $\sigma-$ рациональное число с нечетным знаменателем, $\sigma \geqslant 1$; функции $b_{s j}(t)$ определены и непрерьвны при $t \geqslant 0$ и удовлетворяют неравенствам

$$
\left|b_{s j}(t)\right| \leqslant D(t+1)^{\alpha}
$$

где $D$ и $\alpha$-положительные постоянные.

Применяя теорему 1 , получаем, что при выполнении неравенства (4) нулевое решение системы (7) асимптотически устойчиво.

Покажем, что при некоторых дополнительных ограничениях на функции $b_{s j}(t)$ найденные условия асимптотической устойчивости можно уточнить, используя предложенный в работах [5], [6] метод построения функций Ляпунова для нестационарных систем. 
Пусть при всех $t \geqslant 0$ имеют место неравенства

$$
\left|\int_{0}^{t} b_{s j}(\tau) d \tau\right| \leqslant D_{1}(t+1)^{\beta}
$$

где $D_{1}>0, \beta \geqslant 0$. Учитывая оценку (8), можно считать, что $\beta \leqslant \alpha+1$.

Предположим также, что функция $V(X)$, соответствующая невозмущенной системе (1), является дважды непрерьвно-дифференцируемой. Для этого достаточно, чтобы функции $f_{s}(X)$ были дважды непрерывно-дифференцируемьми (см. [3, с. 119-123]).

ТЕОРема 2. При выполнении неравенства

$$
2 \sigma>(\alpha+\beta)(\mu-1)+\mu+1
$$

нулевое решение системы (7) асимптотически устойчиво.

ДокАЗАТЕЛЬСТво. Функцию Ляпунова для возмущенной системы выберем в виде

$$
V_{1}=V(X)-\sum_{s=1}^{n} \frac{\partial V}{\partial x_{s}} \sum_{j=1}^{k} \int_{0}^{t} b_{s j}(\tau) d \tau h_{j}(X)
$$

Получим, что при всех $t \geqslant 0, X \in E^{n}$ справедливы неравенства

$$
\begin{aligned}
& a_{1}\|X\|^{m}-c_{1}\|X\|^{m+\sigma-1}(t+1)^{\beta} \leqslant V_{1}(t, X) \leqslant a_{2}\|X\|^{m}+c_{1}\|X\|^{m+\sigma-1}(t+1)^{\beta}, \\
& \frac{d V_{1}}{d t} \leqslant-b_{1}\|X\|^{m+\mu-1}+c_{2}\|X\|^{m+\mu+\sigma-2}(t+1)^{\beta}+c_{3}\|X\|^{m+2 \sigma-2}(t+1)^{\alpha+\beta},
\end{aligned}
$$

где $a_{1}, a_{2}, b_{1}, c_{1}, c_{2}, c_{3}$ - положительные постоянные.

Дальнейшее доказательство аналогично доказательству теоремы 1 .

ЗАмечание 1. Если $\beta=\alpha+1$, то условия (4) и (9) совпадают. В случае, когда $\beta<\alpha+1$, теорема 2 уточняет условия асимптотической устойчивости, полученные в теореме 1 .

ЗАмЕчАниЕ 2 . Если $\alpha+\beta<1$, то из неравенства (9) следует, что асимптотическая устойчивость нулевого решения системы (7) может иметь место и при $\sigma \leqslant \mu$.

ПРИмеР 1. Рассмотрим систему

$$
\dot{x}_{s}=\frac{\partial W(X)}{\partial x_{s}}, \quad s=1, \ldots, n .
$$

Здесь $W(X)$ - дважды непрерывно-дифференцируемая отрицательно определенная однородная функция порядка $\mu+1, \mu>1$.

Нулевое решение этой системы асимптотически устойчиво, а однородную функцию Ляпунова можно выбрать в виде

$$
V(X)=\sum_{s=1}^{n} x_{s}^{2}
$$

Пусть задана возмущенная система

$$
\dot{x}_{s}=\frac{\partial}{\partial x_{s}}\left(W(X)+W_{1}(X) t^{1 / 8} \cos \left(t^{1 / 2}\right)+W_{2}(X) t^{1 / 4} \sin t\right), \quad s=1, \ldots, n,
$$

где $W_{1}(X)$ и $W_{2}(X)$ - дважды непрерывно- дифференцируемые однородные функции порядка $\sigma+1, \sigma \geqslant 1$.

Получаем, что $\alpha=1 / 4, \beta=5 / 8$. Таким образом, условия асимптотической устойчивости нулевого решения системы $(10)$ имеют вид $\sigma>(15 \mu+1) / 16$. 
4. Предположим теперь, что интегралы

$$
\int_{0}^{+\infty} b_{s j}(\tau) d \tau
$$

сходятся. В этом случае можно считать, что $\beta=0$. Используя теорему 2 , находим достаточные условия асимптотической устойчивости нулевого решения системы (7):

$$
2 \sigma>\alpha(\mu-1)+\mu+1 .
$$

Эти условия можно уточнить, выбирая при доказательстве теоремы 2 функцию Ляпунова в виде

$$
V_{1}=V(X)+\sum_{s=1}^{n} \frac{\partial V}{\partial x_{s}} \sum_{j=1}^{k} \int_{t}^{+\infty} b_{s j}(\tau) d \tau h_{j}(X) .
$$

Получим, что имеют место теоремы.

Теорема 3. Если $2 \sigma=\alpha(\mu-1)+\mu+1$, то нулевое решение системы (7) асимптотически устойчиво.

ТЕОРема 4. Пусть при всех $t \geqslant 0$ справедливы неравенства

$$
\left|\int_{t}^{+\infty} b_{s j}(\tau) d \tau\right| \leqslant D_{1}(t+1)^{\beta},
$$

определяющие скорость сходимости интегралов (11). Здесь $D_{1}>0, \beta<0$. Тогда при выполнении неравенства (9) нулевое решение системы (7) асимптотически устойчиво.

ЗАмЕчАниЕ 3. Если $\alpha+\beta<-1$, то из условия (9) следует, что асимптотическая устойчивость имеет место при всех $\mu$ и $\sigma$, удовлетворяющих неравенствам

$$
\mu>1, \quad \sigma \geqslant 1 \text {. }
$$

Покажем далее, что в случае, когда $-1 \leqslant \alpha+\beta<0$, утверждение теоремы 4 можно усилить.

Рассмотрим функции

$$
c_{s j}^{(r i)}(t)=b_{r i}(t) \int_{t}^{+\infty} b_{s j}(\tau) d \tau, \quad r, s=1, \ldots, n, \quad i, j=1, \ldots, k .
$$

Теорема 5. Если функиии $c_{s j}^{(r i)}(t)$ стремятся $\kappa 0$ при $t \rightarrow+\infty$, то при выполнении неравенств (13) нулевое решение системы (7) асимптотически устойчиво равномерно по $t_{0} u X_{0}$.

ДокАЗАтЕЛЬСтво. Рассмотрим функцию $V_{1}(t, X)$, построенную по формуле (12). При всех $t \geqslant 0$ и $X \in E^{n}$ имеем

$$
\begin{gathered}
a_{1}\|X\|^{m}-g_{1}(t)\|X\|^{m+\sigma-1} \leqslant V_{1}(t, X) \leqslant a_{2}\|X\|^{m}+g_{1}(t)\|X\|^{m+\sigma-1}, \\
\frac{d V_{1}}{d t} \leqslant-b_{1}\|X\|^{m+\mu-1}+g_{2}(t)\|X\|^{m+\mu+\sigma-2}+g_{3}(t)\|X\|^{m+2 \sigma-2},
\end{gathered}
$$

где $a_{1}, a_{2}, b_{1}$ - положительные постоянные, а неотрицательные функции $g_{1}(t), g_{2}(t)$ и $g_{3}(t)$ непрерьвны при $t \geqslant 0$ и стремятся к 0 при $t \rightarrow+\infty$.

Используя эти неравенства, дальнейшее доказательство проводится так же, как и доказательство теоремы 5 в работе [7, §4, с. 83-85]. 
ПРимеР 2. Пусть задана система

$$
\dot{x}_{s}=\frac{\partial}{\partial x_{s}}\left(W(X)+W_{1}(X) t \sin \left(t^{7 / 2}\right)\right), \quad s=1, \ldots, n,
$$

где функции $W(X)$ и $W_{1}(X)$ обладают свойствами, указанными в примере 1.

В данном случае $\alpha=1, \beta=-3 / 2$. Следовательно, по теореме 5 получаем, что нулевое решение рассматриваемой системы асимптотически устойчиво равномерно по $t_{0}$ и $X_{0}$.

5. Предложенньй метод построения функций Ляпунова для нестационарных систем можно применять и для получения достаточных условий неустойчивости.

Пусть нулевое решение системы (1) неустойчиво, причем существует функция $V(X)$, обладающая свойствами:

1) функция $V(X)$ не является знакопостоянной отрицательной;

2) $V(X)$ - однородная функция порядка $m, m \geqslant 1$;

3) функция $V(X)$ дважды непрерывно-дифференцируема;

4) производная функции $V(X)$ в силу системы (1) положительно определена.

Условия существования функций такого вида получены в работах [2], [8].

По-прежнему считаем, что интегралы (11) сходятся.

ТЕОРема 6. Если функиии $c_{s j}^{(r i)}(t)$ ограничены при $t \geqslant 0$, то при выполнении неравенства $2 \sigma>\mu+1$ нулевое решение системы (7) неустойчиво.

Для доказательства теоремы снова рассмотрим построенную по формуле (12) функцию $V_{1}(t, X)$. Легко показать, что указанная функция удовлетворяет всем условиям первой теоремы Ляпунова о неустойчивости (см. [3, с. 13]).

СлЕДСТВИЕ. Если функиии $c_{s j}^{(r i)}(t)$ npu $t \rightarrow+\infty$ стремятся $\kappa 0$, mo при выполнении неравенства $2 \sigma \geqslant \mu+1$ нулевое решение системы (7) неустойчиво.

\section{СПИСОК ЦИТИРОВАННОЙ ЛИТЕРАТУРЫ}

[1] Малкин И.Г. Теорема об устойчивости по первому приближению // Докл. АН СССР. 1951. Т. 76. №6. С. 783-784.

[2] Красовский Н. Н. Об устойчивости по первому приближению // ПММ. 1955. Т. 19. № 5. C. $516-530$.

[3] Зубов В.И. Устойчивость движения. М.: Высшая школа, 1973.

[4] Барбашин Е. А. Функции Ляпунова. М.: Наука, 1970.

[5] Александров А. Ю., Прасолов С. А. Об устойчивости систем линейных дифференциальных уравнений с рекуррентными коэффициентами // Изв. РАН. Теория и системы управления. 1995. № 3. С. 8-14.

[6] Александров А. Ю. Об устойчивости решений некоторого класса нелинейных нестационарных дифференциальных уравнений // Дифференциальные уравнения и их приложения. Материалы Международной конференции (22-24 декабря 1994г.). Саранск: Изд-во Мордовского ун-та, 1995. С. $126-132$.

[7] Зубов В. И. Колебания и волны. Л.: Изд-во ЛГУ, 1989.

[8] Каневский А.Я., Рейзинь Л. Э. Построение однородных функций Ляпунова-Красовского // Дифференц. уравнения. 1973. Т. 9. № 2. С. 251-259. 